ÉGYPTE

monde arabe

\section{Égypte/Monde arabe}

4 | 2007

Figures de la santé en Égypte

\title{
Santé et hygiénisme dans les villes du canal de Suez
}

Fin $19^{\mathrm{e}}$ siècle - $1^{\text {ère }}$ moitié du $20^{\mathrm{e}}$ siècle

\section{Céline Frémaux}

\section{(2) OpenEdition}

Journals

Édition électronique

URL : https://journals.openedition.org/ema/1759

DOI : 10.4000/ema.1759

ISSN : 2090-7273

Éditeur

CEDEJ - Centre d'études et de documentation économiques juridiques et sociales

Édition imprimée

Date de publication : 31 décembre 2007

Pagination : 75-101

ISBN : 2-6905838-43-4

ISSN : 1110-5097

Référence électronique

Céline Frémaux, "Santé et hygiénisme dans les villes du canal de Suez », Égypte/Monde arabe [En

ligne], 4 | 2007, mis en ligne le 31 décembre 2008, consulté le 07 juillet 2022. URL : http://

journals.openedition.org/ema/1759; DOI : https://doi.org/10.4000/ema.1759 


\section{SANTÉ ET HYGIÉNISME DANS LES VILLES DU CANAL DE SUEZ}

(FIN xIX ${ }^{\mathrm{e}}$ SIÈCLE - 1 $^{\text {è }}$ MOITIÉ DU xx ${ }^{\mathrm{e}}$ SIÈCLE)

L es villes du canal de Suez, fondées ou développées à partir du percement de l'isthme, sont des villes égyptiennes atypiques. À la fois lieux de travail et de commerce, lieux de vie et de sociabilité, mais aussi espaces de représentation de la Compagnie universelle du canal maritime de Suez, elles présentent un aspect polymorphe reflétant la diversité de leur fonction et la mixité de leur population. Toutes, à travers I'histoire de leur conception et de leur développement, attestent de la difficulté d'implanter et de maintenir une population dans cette région, enjeu vital de la réussite de l'entreprise.

Comme dans tous les cas d'implantations d'Européens dans les pays du sud de la Méditerranée ou dans les pays tropicaux, que ce soit dans le cadre d'entreprises commerciales ou d'entreprises de colonisation, la difficulté d'adaptation au climat et de résistance aux maladies locales a entraîné la mise en place de dispositifs spécifiques visant à assurer confort et hygiène à la maind'œuvre étrangère.

Cet article n'entend pas faire un bilan des morts et des malades pendant le chantier, pas plus qu'une histoire sociale de l'entreprise. Il a pour objectif d'identifier, d'après les archives de la Compagnie, les problématiques relatives aux enjeux sanitaires qui se sont fait jour dans les villes développées sur les rives du canal de Suez. Évidemment, les maladies sur le chantier étaient nombreuses, surtout avant la réalisation du canal d'eau douce. L'hépatite, la dysenterie, les embarras gastriques et l'ophtalmie étaient les plus courantes, sans compter les épidémies qui ont sévi à plusieurs reprises telles que le typhus, la dysenterie et surtout le choléra en 1865 (Montel, 1998, p. 206217). Le nombre d'ouvriers égyptiens morts des suites de ces maladies ou de la pénibilité des travaux reste un sujet de débat, lié au manque de fiabilité des sources accessibles. Si l'exactitude des chiffres cités dans les archives de la Compagnie peut être mise en cause, considérant que les comptes rendus du service de santé devaient alimenter les bilans de l'entreprise destinés 
aux actionnaires, la véracité des discours véhiculés par la presse anglaise de l'époque est tout autant sujette à caution. II est également probable qu'il faille revoir à la baisse les chiffres diffusés par les manuels scolaires d'après la nationalisation de 1956. À l'appui de cette hypothèse, il est intéressant de signaler qu'une thèse égyptienne, parue en 1951, sur les préjudices économiques causés par l'entreprise de percement du Canal, ne fait aucunement mention de nombreux fellahs morts pendant le creusement (El Hefnaoui dans Jagailloux 1986, p. 102). D'autre part, on peut, avec Serge Jagailloux, refuser de mettre en doute l'honnêteté intellectuelle des membres du corps médical, de nationalités diverses, à l'origine des bilans de santé de la Compagnie, eux-mêmes victimes des maladies qu'ils tentaient d'enrayer (quatre médecins sur les onze en exercice pendant les travaux ont trouvé la mort). Dans l'état actuel des sources consultées, il semble vain de s'attarder sur ce sujet et préférable de se pencher sur les fondements et les moyens d'action des dispositifs de santé dans l'isthme.

À partir de l'installation des premiers campements, comment la Compagnie s'efforce-t-elle de garantir la bonne situation sanitaire des villes du Canal si ce n'est par la mise en œuvre d'une organisation efficace et de méthodes déjà éprouvées en Europe? De la surveillance sanitaire aux soins, de nombreux domaines d'action sont confiés aux médecins. Ce sont d'abord eux, présents en effet dès l'ouverture du chantier de percement du Canal en 1859, qui sont chargés de préserver et de garantir la santé publique et privée. L'organisation d'un service très vite doublé de la création d'un réseau médical dense a conduit Nathalie Montel à poser la question de savoir si la Compagnie n'était pas un État dans l'État (Montel, 1998, p. 171). Mais s'il est vrai que pendant la période de creusement la Compagnie est la seule à gérer les questions sanitaires, dès l'ouverture du Canal les responsabilités sont partagées dans la plupart des domaines, qu'il s'agisse de surveillance des épidémies, de soins ou encore de questions urbanistiques.

L'originalité de l'activité du personnel médical dans I'isthme est liée aux particularités des maladies qui sévissent sous le climat méditerranéen, alors qu'elles ont plus ou moins disparu d'Europe occidentale. Avant les soins, ce sont bien les études, les actions préventives et l'assainissement qui préoccupent les médecins. Ils ont à cœur d'appliquer sur place les théories hygiénistes qui ont contribué à réduire sensiblement en Europe les épidémies telles que la peste, le paludisme ou le choléra. La Compagnie contribue, en relation avec les services du gouvernement égyptien, à la lutte contre ces fléaux en finançant l'organisation des services de quarantaine et le contrôle des navires de passage, des campagnes de prévention et des missions exploratoires sur les origines de ces maladies.

Mais au-delà de ces actions directes, la lutte contre les maladies et les épidémies, largement influencée par la théorie climatique des maladies et l'hygiénisme, concerne également le domaine de la construction. En France, deux corps de fonctionnaires d'État ont directement affaire à l'hygiène des villes à la 
fin du XIX $x^{\mathrm{e}}$ siècle : les médecins militaires, observateurs des villes de garnison, et les ingénieurs des Ponts et Chaussées, qui dirigent depuis longtemps les services techniques parisiens (Claude, 1999, p. 272). Le trio médecin/ingénieur/architecte règne alors sur le développement des villes en Europe. Cette organisation migre d'ailleurs en Égypte sous le règne de Muhammad 'Alî. En effet, le Conseil de Tanzîm du Caire, créé en 1843 pour " l'embellissement de la capitale et l'amélioration et le redressement de ses rues », est composé d'ingénieurs et de médecins, pour moitié européens. On compte parmi eux le médecin et président du conseil de Santé Clot Bey (Volait, 2005, p. 88). Ce type $\mathrm{d}^{\prime}$ association perdurera dans la première moitié $d u x^{\mathrm{e}}$ siècle. Gustave Kahn écrit en 1901 : "Actuellement I'hygiène est Dieu, le médecin son prophète, et l'architecte obéit à leurs prescriptions» (p. 299). Cette relation d'affinité est d'autant plus prégnante en France qu'une chaire d'hygiène existe à l'École spéciale d'architecture. Emile Trélat, directeur de l'école, déclare dans son discours d'ouverture de 1900 :

Le médecin, l'architecte et l'ingénieur, sont, chacun dans sa sphère de compétence, les metteurs en scène des prescriptions de I'hygiène. À l'architecte et à l'ingénieur échoient l'ordonnance des habitations et l'entretien du territoire commun. (cité dans Mory, 2001, p. 149)

C'est exactement cette association qui préside à I'aménagement du territoire de la zone du canal de Suez. Des médecins employés par la Compagnie, dans une perspective d'explication mésologique des maladies, étudient les conditions sanitaires et le climat des emplacements des camps d'ouvriers. En cernant ainsi l'influence exercée par le milieu sur la santé, ils orientent le travail des ingénieurs, pour la plupart issus de l'École des Ponts et Chaussées, en matière d'assainissement, d'urbanisme et de construction.

\section{LES ENJEUX DES SERVICES DE SANTÉ DANS L'ISTHME}

Le service de santé de la Compagnie est créé dès 1859. Devenu un organe habituel des grandes entreprises françaises, il est considéré indispensable dans l'isthme pour éviter une hécatombe telle que celle déclenchée par le creusement du canal Mahmûdiyya en 1838 (Jagailloux, 1986, p. 96). Au-delà de la survie et de la santé des ouvriers et des fellahs employés pour les travaux de percement, garantir la salubrité de la zone du Canal correspond pour la Compagnie à des enjeux multiples, au premier rang desquels figure l'intérêt économique. La santé des employés a en effet des répercussions directes sur le bon fonctionnement de l'entreprise. D'autre part, il est nécessaire, à la fois pour attirer des travailleurs européens et pour valoriser les nouvelles cités, de gommer les a priori négatifs sur l'état sanitaire de la zone du Canal. Enfin, la Compagnie et le gouvernement égyptien sont tenus, sous la pression internationale, d'assurer la lutte contre les épidémies. 


\section{Un enjeu majeur : le coût de la santé}

Dans Le Canal de Suez, bulletin décadaire de la Compagnie, organe d'information et de liaison des employés, les travaux d'amélioration des villes du Canal sont souvent évoqués. Le conseil d'administration rappelle ainsi que "La santé et le bien-être de nos courageux et dévoués collaborateurs dans l'isthme sont [...] un sujet de légitime préoccupation » (Le Canal de Suez, 16 mai 1888). Quels sont les fondements de cette préoccupation ? Tout d'abord, lors de la concession cédée par le vice-roi d'Égypte à la Compagnie, certaines exigences relatives à la santé ont été imposées. Le règlement du 20 juillet 1856 concernant l'emploi des ouvriers stipule :

La Compagnie sera tenue d'abriter les ouvriers, soit sous des tentes, soit dans des hangars ou maisons convenables. Elle entretiendra un hôpital et des ambulances, avec tout le personnel et le matériel nécessaire pour traiter les malades à ses frais. Chaque ouvrier malade recevra à l'hôpital ou dans les ambulances, outre les soins que réclame son état, une paye d'une piastre et demie pendant tout le temps qu'il ne pourra pas travailler. (Vaultier, 1956, p. 1)

Cette préfiguration d'un système d'assurance maladie est remarquable à cette époque. Mais la Compagnie elle-même connaissait tout l'intérêt qu'il y avait à soigner et surtout à prévenir les maladies de ses employés.

Le médecin en chef de la Compagnie, le Dr Aubert-Roche, nommé dès 1859 par Ferdinand de Lesseps, s'exprime de manière directe à cet égard. II ne fait entrer en ligne de compte ni sentiment de charité ni service humanitaire. L'objectif principal de sa mission est purement économique, comme il l'écrit dans son rapport sur l'organisation du service de santé : «Au nom de l'intérêt de la Compagnie, la santé des travailleurs est un bénéfice, non seulement au point de vue du travail en plus, mais par rapport à la facilité de se procurer des travailleurs et à la colonisation de l'Isthme, par conséquent au prix des journées et de la valeur des terrains » (CAMT, 1859a). Dans le même rapport, AubertRoche explicite la mission du service médical dans l'isthme en l'opposant aux expériences vécues dans certaines colonies françaises, notamment en Algérie :

En principe, le service de santé qui doit être installé dans I'Isthme de Suez ne ressemble pas aux services qui existent soit dans l'armée, soit dans les chemins de fer, soit dans toute autre grande Compagnie. Là le médecin n'est appelé que dans un but, guérir la maladie lorsqu'elle s'est déclarée, et la direction de ce service est généralement sous les ordres d'une personne totalement étrangère à la science médicale. Ainsi, pendant longtemps, en Algérie, la Direction de la santé était confiée à un conseil composé d'officiers du génie d'artillerie et même de cavalerie. Ce beau système a coûté des millions à la France, et des milliers d'hommes ont été enlevés par les fièvres et la dysenterie. L'Algérie a été regardée comme insalubre et cette prétendue insalubrité a contribué pour beaucoup à arrêter l'émigration et la colonisation de ce pays. (Id. p. 11) 
Le service médical de la Compagnie doit donc, condition nécessaire à la réussite économique du projet, contrer les nombreux préjugés existants sur l'insalubrité de la zone du Canal.

Les médecins de la Compagnie œuvrent en premier lieu à la connaissance de la topographie et du climat de l'isthme. Les rapports hebdomadaires du médecin de Port-Saïd mentionnent les conditions météorologiques, les problèmes de santé et les conditions sanitaires du camp. Pour chaque cas de consultation, I'auteur précise également si les malades sont en arrêt de travail ou s'ils risquent de l'être, établissant un lien direct entre la salubrité et le coût de la santé (CAMT, 1859b). En Europe, les topographies médicales, apparues à la fin du $\mathrm{xVIII}^{\mathrm{e}}$ siècle ${ }^{1}$ et visant à établir la corrélation entre les maladies et les conditions environnementales et climatiques en un lieu donné, déclinent au XIX ${ }^{\mathrm{e}}$ siècle au profit de l'explication sociale des maladies (Barles, 1999, p. 7). Les travaux des médecins Claude Lachaise et Louis-René Villermé sur Paris s'orientent en effet vers les causes d'insalubrité propres à la ville (mode de construction, règlement de voirie, etc.) (voir Fijalkow, 1998, $1^{\text {ère }}$ partie). Dans des sites lointains et inhabités comme celui des futures villes du Canal, le type d'étude traditionnel perdure tant que l'étiologie des maladies tropicales reste inconnue. Les limites du genre de la topographie médicale vont cependant pousser les médecins à explorer d'autres méthodes. Nous allons voir que le service de santé de la Compagnie a participé aux progrès de la science médicale et les a même stimulés.

\section{Imposer de nouvelles représentations de la santé}

Le peuplement des villes du Canal, comme pour tout territoire éloigné de la métropole, est considéré comme difficile en raison des rudes conditions de vie dans les zones d'implantation. Les représentations négatives de la santé et de I'hygiène dans ces territoires sont particulièrement tenaces. II est vrai que l'introduction en Égypte d'une nouvelle conception de la santé publique, appuyée par le médecin français Clot-bey, appelé par le vice-roi Muhammad 'Alî en 1825, est récente et que les principes de l'hygiène n'ont pas encore pénétré en profondeur (Chiffoleau, 2001, p. 393). L'écart entre pratiques hygiénistes européennes et usages locaux est commun à bien des situations outre-mer, telle que celle décrite par Gabriel Garcia Marquez dans L'Amour aux temps du choléra. Le romancier relate l'expérience vécue par Juvénal Urbino, médecin formé à Paris à la fin du XIXe siècle, confronté à ce

1. La Société Royale de Médecine, fondée en 1776, lance l'élaboration « d'un plan topographique et médical de la France, par lequel le tempérament, la constitution et les maladies des habitants de chaque province ou canton seraient considérés relativement à la nature et à l'exposition du sol ». 
décalage à son retour dans sa petite ville des Caraïbes (Garcia Marquez, 1987, p. 142).

Mais ce sont surtout les préjugés véhiculés par la littérature, les relations de voyages en Orient et même par certains traités théoriques qui contribuent à entretenir ces représentations. Les architectes Albert Lenoir et Pierre Landry, dans leur ouvrage Théorie des villes. Comment les villes se sont formées, devant servir de base à des projets d'embellissement de la ville de Paris, décrivent les villes égyptiennes. Parmi les griefs relatifs à leur insalubrité, ils attribuent aux habitants la responsabilité des pires maux : "On fit pis encore, on tira hors de chez soi le cadavre de l'animal mort dans l'étable ou dans l'écurie, il se décomposa en pleine rue ; c'est le fellah, paysan de l'Égypte, ramenant périodiquement la peste par cette incurie » (Lenoir et Landry, 1854, p. 297). De tels propos ont nourri le sentiment de méfiance à l'égard des conditions hygiéniques dans les pays du sud de la Méditerranée.

Afin d'y remédier et d'attirer la main-d'œuvre étrangère, les médecins chargés de la surveillance de l'hygiène publique s'efforcent d'assurer en priorité la propreté des villes. Ils font nettoyer les rues, les maisons, les cloaques, brûler les immondices, vider les lieux d'aisance, parquer les porcs, etc. Le médecinchef de la Compagnie souligne d'ailleurs que «bien des villes en France sont loin d'être aussi bien tenues et que Port-Saïd en Europe se nommerait une ville très propre »(CAMT, 1865a). De même, il fait remarquer que peu de villes en France sont aussi propres et aussi salubres qu'Ismaïlia. Enfin, il démontre le rapport de cause à effet entre salubrité et santé en dressant un bilan démographique démonstratif : "La mortalité des arabes dans I'Isthme est d'un tiers moindre qu'en Égypte ». Ce rapport est encore attesté par le défenseur du projet d'installation d'un hôpital anglais à Port-Saïd :

On ne saurait prétendre qu'il y a la malaria ici ou que cet endroit est un foyer exhalant l'épidémie. La fièvre typhoïde, résultat naturel de la saleté, du manque de propreté et d'une population excessive, est presque inconnue parmi les habitants et pas un cas parmi vingt de cette maladie n'est engendré dans la ville. Pendant les six derniers mois, tous les cas de typhus résultaient du trafic maritime. Huit cas qui se sont terminés de manière fatale en 1882 venaient tous de Damiette. II ne s'est pas produit un seul cas local ici quoique le typhus ait emporté des milliers de personnes dans presque chaque autre ville de la Basse Égypte. ( La commémoration nationale de la mémoire de Gordon », The Shipping Gazette, 20 avril 1885)

\section{Le Canal, vecteur de propagation des épidémies?}

Une autre raison majeure de veiller à la situation sanitaire de l'isthme tient aux craintes suscitées par le percement du Canal. Les Européens se défient de ce passage ouvert aux épidémies de choléra venant de l'Inde ou de l'Arabie (CAMT, 1869). Avant les découvertes de l'origine microbienne de ces maladies, 
la doctrine du contagionnisme, fondée sur la transmission par contact de la peste, de la fièvre jaune et du choléra, impose la plus grande prudence. La peur des «épidémies d'origine asiatique», que l'accélération des liaisons entre l'Orient et I'Occident ranime, est perceptible à la lecture des rapports des conférences sanitaires internationales qui, à partir de 1851, s'attachent à I'organisation du service sanitaire en Orient (voir Rasmussen, 2001, p. 213). Le littoral de la mer Rouge fait particulièrement I'objet des préoccupations en raison du passage des pèlerins au retour de la Mecque et de la navigation dans le canal de Suez. Les congrès internationaux d'hygiène qui se succèdent à partir de 1852 tentent quant à eux de définir les meilleures conditions de protection sans entraver la forte croissance du trafic via le Canal (Pinol, 2003, p. 126). Ainsi la lutte internationale contre les fléaux épidémiques se structure-t-elle peu à peu. Entre autres réalisations, elle aboutit, à l'occasion de la conférence de Venise de 1892, à la suppression du régime spécial de passage en quarantaine des seuls navires anglais, étendant cette facilité à tout navire "sain » et généralisant au contraire les mesures sérieuses à tout navire suspect sans exception de nationalité. En 1907, un arrangement international est signé à Rome pour la création à Paris d'un Office international d'hygiène publique (CAMT, 1938). L'Égypte figure, au même titre que la plupart des États européens, les États-Unis et le Brésil, parmi les gouvernements ayant ratifié I'arrangement. L'Office a pour objectif de recueillir et de porter à la connaissance des États participants les faits et les documents d'un caractère général qui intéressent la santé publique, spécialement en ce qui concerne les maladies infectieuses, notamment choléra, peste et fièvre jaune.

\section{Le service de quarantaine, un contrôle médical nécessaire}

La première mesure à prendre pour garantir la sécurité sanitaire de la zone du Canal et, au-delà, de l'Europe entière, est de contrôler les passages et d'organiser les quarantaines. Jusqu'en 1831, l'Égypte comptait quelques stations quarantenaires à Alexandrie, Damiette, Rosette et Choubra. L'épidémie de choléra de 1831 suscita la mise en place d'une Commission consulaire de santé qui devint quelques années plus tard I'Intendance de santé (Jagailloux, 1986, p. 60-81).

Dès 1864, Port-Saïd est soumise au régime quarantenaire. Par nécessité, la Compagnie et le gouvernement s'entendent sur les conditions de fonctionnement du service de quarantaine dans le Canal. L'agence sanitaire de Damiette dont relève Port-Saïd prend en charge le salaire des marins employés pour la conduite des barques tandis que les logements et les barques sont mis à disposition en pure libéralité par la Compagnie (CAMT, 1864). Un lazaret provisoire, permettant $d$ 'isoler les malades au besoin, est installé par la Compagnie dans une maison située à l'écart de la ville, " où l'air pur et vivifiant de la mer arrive sans obstacle»(CAMT, 1866). Des lazarets définitifs seront construits par le conseil 
sanitaire d'Égypte à Port-Saïd et Port-Tewfik, dans les conditions requises par la Convention de Venise. La Compagnie participera à la dépense de ces constructions de manière à pouvoir, le cas échéant, y placer ses pilotes (CAMT, 1899a).

La surveillance sanitaire autour du Canal est rigoureuse. Les cadres de la Compagnie prennent régulièrement des mesures pour empêcher tout contact entre les passagers à bord des navires et les habitants. Des mesures quarantenaires sont régulièrement prises dans le but d'empêcher les rapprochements entre les ouvriers et les arabes nomades ou les caravanes venant d'Asie qui n'auraient pas purgé leur quarantaine. Ainsi en est-il en 1875 lorsque des symptômes d'épidémie cholérique sont signalés en Syrie, en 1878 lorsqu'une épidémie est déclarée dans la province du Hedjaz, ou encore en 1879 lorsque la peste est signalée en Russie et le typhus en Roumélie (CAMT, 1883). Les durées d'isolement sont fixées par le Conseil sanitaire maritime et quarantenaire. La Compagnie pâtit évidemment de ces temps d'arrêt des bateaux qui apportent approvisionnement et matériel nécessaires aux ateliers. Mais des arrangements sont parfois trouvés. Ainsi, lorsqu'un début d'épidémie de choléra se déclare en 1883, I'intendance sanitaire d'Alexandrie autorise, à la demande de la Compagnie, l'expédition des approvisionnements les plus indispensables pour le fonctionnement des usines hydrauliques d'Ismaïlia et de Suez et des chantiers de curage du Canal, à certaines conditions : escorte de gardes sanitaires payés par la Compagnie, interdiction de communication des équipages avec les points du Canal non contaminés, quarantaine des marchandises débarquées et des équipages (Ibid.).

Les flux de pèlerins de retour de la Mecque n'échappent pas aux mesures d'isolement. Le convoyage des navires à pèlerins est assuré par un remorqueur ayant à son bord des soldats chargés de la surveillance, et des matelots de la Compagnie qui assurent les manœuvres. De plus, le gouvernement fait établir rive asiatique, de Suez à Port-Saïd, des postes militaires composés chacun de huit hommes montés à dromadaire, destinés à former un cordon sanitaire pour s'opposer au passage en Afrique des pèlerins venant du Hedjaz qui tenteraient de se soustraire aux mesures quarantenaires (CAMT, 1890). Les soldats ont même l'ordre de tirer sur tout contrevenant.

Si les adversaires du projet de percement dénonçaient l'importance des risques épidémiques, force est de constater que le gouvernement et la Compagnie ont conjugué leurs efforts pour leur donner tort.

\section{ORGANISATION DES SERVICES DE SANTÉ AUTOUR DU CANAL}

La préoccupation majeure de la Compagnie pour les questions sanitaires se traduit dans l'organisation de son service de santé. Administré comme les autres services, de celui des travaux à celui du transit, il dépend tour à tour de l'agence supérieure des travaux ou de la direction générale des travaux. En plus d'une dizaine de médecins et pharmaciens, il comprend un important personnel 
subalterne de nationalités diverses, des infirmiers aux aides médecins. Outre les salaires du personnel, le budget de ce service couvre les importants équipements médicaux de toute nature, des ambulances aux hôpitaux. Le service de santé intervient dans des domaines aussi divers et complémentaires que le contrôle du transit décrit ci-dessus, l'implantation d'un véritable réseau médical et même de recherche.

\section{Le réseau médical de la Compagnie}

Le service de santé de la Compagnie met en effet en place dans l'isthme un réseau médical dense organisé, aux dires de ses concepteurs, « comme en Europe ».

Les deux premiers services de médecine sont installés à Port-Saïd et à Timsah. Leur organisation distingue un service sanitaire chargé de la prévention des maladies et un service médical chargé de la guérison. À l'ouverture du chantier, trois centres sont prévus pour le canal maritime et un centre pour celui d'eau douce avec chacun un hôpital prévu pour cinquante personnes.

À la fin de la période de creusement du Canal, le service de santé est remis au gouvernement égyptien. La Compagnie continue cependant à œuvrer dans ce domaine en construisant de nouveaux établissements destinés à accueillir ses employés et à compléter les dispositifs existants. La construction d'un hôpital à Port-Saïd est en effet envisagée en 1884 car l'hôpital du gouvernement n'est plus en rapport avec l'importance de la population de la ville (CAMT, 1884). Cette dernière a quasiment doublé en moins de quinze ans, passant de 10000 habitants en 1869 à 17000 en 1883 (Crosnier-Leconte, 2006, p. 9 ; Baedeker, 1903). Toutefois, le programme des travaux d'amélioration du Canal conduit à revoir la question de son emplacement. Un rapport du 18 octobre 1885 préconise son installation au chantier numéro VI, à proximité du Canal et sur un branchement à créer de la route d'Ismaïlia au chalet du vice-roi. Des raisons sanitaires confortent ce choix, les conditions hygiéniques y étant reconnues meilleures qu'à Port-Saïd (CAMT, 1885). Conçu et développé en phase avec les progrès de la science médicale, l'hôpital Saint-Vincent-de-Paul à Ismailia est un témoin de l'évolution de l'architecture hospitalière aux $x_{1 x}{ }^{\mathrm{e}}$ et $\mathrm{xx}^{\mathrm{e}}$ siècles. Les premiers pavillons, des constructions à rez-de-chaussée ou à un étage éclatées autour d'espaces verts, sont représentatifs des hôpitaux hygiénistes du xix ${ }^{\mathrm{e}}$ siècle pour la construction desquels primait le souci d'une bonne aération. C'est un hôpital pavillonnaire tel qu'il se conçoit après la révolution pasteurienne, où les méthodes de l'asepsie et de l'antisepsie peuvent être appliquées rigoureusement (Foucart, 1981, p. 43-52). Les plans du nouveau pavillon de chirurgie préparés par l'architecte Paul Nelson en 1932 marquent quant à eux un autre tournant de l'architecture hospitalière (CAMT, 1932). Il s'agit d'un immeuble de quatre niveaux, dans lequel la répartition des services permet des économies de parcours au personnel médical. Dans le domaine de la construction hospitalière, la Compagnie fait appel aux meilleurs 
spécialistes. Déjà connu à l'époque pour son projet de cité hospitalière de vingt-six étages à Lille, Paul Nelson deviendra en effet l'un des architectes les plus influents dans l'évolution de l'architecture des hôpitaux au xx siècle et I'hôpital mémorial de Saint-Lô constituera à partir des années 1950 une référence (Duval, 1981, p. 59-65).

En dehors de I'hôpital Saint-Vincent, la Compagnie conserve à Ismaïlia son médecin attitré, qui donne gratuitement des soins au personnel. À PortSaïd, la Compagnie négocie un système d'abonnement avec quatre médecins sur place (CAMT, s.d.2). Le réseau médical comprend en outre de nombreux dispensaires, pharmacies et cabinets de spécialistes, du dentiste à l'ophtalmologiste. Les soins, qu'ils soient donnés à domicile, dans les dispensaires ou à I'hôpital, sont assurés gratuitement pour tout le personnel de la Compagnie. Le bénéfice des soins s'étendra, au fur et à mesure de l'avancée du peuplement des villes du Canal, aux familles et aux domestiques (CAMT, 1901). Les tarifs d'admission sont calculés en fonction des revenus et revus régulièrement. Dans ce domaine, la Compagnie a fait figure en Égypte de "précurseur ». On sait que ce n'est qu'en 1953 qu'un arrêté égyptien accordera tous les soins médicaux aux salariés des entreprises d'au moins cinquante travailleurs (Monnerot-Dumaine, 1956, p. 7).

Un article d'une revue publiée par le ministère égyptien des Questions sociales en 1952 rend hommage aux initiatives précoces de la Compagnie en matière de questions médicales et sociales :

Rien ne saurait être plus utile et plus encourageant que d'acter les progrès réalisés par des entreprises extragouvernementales, utile pour ceux à qui ils peuvent servir d'exemple, encourageant pour ceux qui en sont les auteurs. [...] La Compagnie du canal avait prévu, dès l'origine et bien avant que les législations sociales aient songé dans le monde à mettre les entreprises en devoir d'assurer les soins médicaux aux membres de leur personnel, l'installation d'un hôpital, de cliniques dans les villes du Canal. (al-mujtama' al-jadîd, décembre 1952)

\section{La lutte contre les épidémies, un tremplin pour les progrès de la science médicale}

La Compagnie entreprend une lutte acharnée contre les fléaux qui sévissent dans cette partie du monde. Les épidémies sont combattues non seulement une fois qu'elles sont déclarées, par la quarantaine ou l'administration de soins, mais aussi par des soins préventifs ou des recherches sur les origines des maladies.

Ainsi les étapes de l'assainissement d'Ismaïlia sont-elles liées à la lutte contre le paludisme. André Pressat, médecin de la Compagnie et directeur de I'hôpital Saint-Vincent résume les débuts difficiles de la ville, confrontée à I'un des fléaux des pays chauds tant redoutés : "Ismaïlia a été bâtie il y a 
trente ans. Elle n'a donc pas de passé, mais elle a une histoire, et son histoire est toute entière celle du paludisme » (Pressat, 1904).

De sa fondation en 1862 à l'apparition des fièvres en 1877, la ville était saine. Ismaïlia était considérée comme une oasis de verdure que sa ceinture de déserts avait jusque-là préservée des influences miasmatiques de la BasseÉgypte. Les relevés des médecins montrent que les cas de fièvre sont apparus à partir de 1877, soit quinze ans après la fondation de la ville, et n'ont dès lors pas cessé avant le début du xx $x^{\mathrm{e}}$ siècle, avec des pics en 1886, 1891 et 1900. Les fièvres de 1877 marquent les premières difficultés du peuplement d'Ismaïlia. Le constat en 1878 est grave :

La maladie a perdu son caractère primitif. Elle était épidémique, elle est devenue endémique. Elle était générale, longue et bénigne, elle est devenue beaucoup plus rare, courte et mortelle. Le moral de votre personnel d'Ismaïlia est gravement atteint. [...] La mort dans un lieu solitaire comme Ismaïlia est particulièrement lugubre. (CAMT, 1878)

Suivant l'avis des médecins, les ingénieurs s'attachent d'abord à supprimer les causes d'humidité du sol et d'infiltrations dans le sous-sol. Le canal de ceinture et les vastes cultures maraîchères sont abandonnés, les marais comblés (CAMT, s.d.1). Les hygiénistes s'élèvent contre la végétation et les cultures. Le directeur des services sanitaires d'Égypte dénonce, notamment en 1892, I'effet néfaste provoqué par le rideau formé par la végétation faisant obstacle à la circulation d'air. Des trouées sont alors percées dans les arbres pour y remédier. Malgré tous les efforts, la fièvre continue à sévir. D'autres causes évoquées pour l'apparition des fièvres paludéennes ont trait aux travaux de canalisation effectués autour d'Ismaïlia. L'apparition des premières fièvres serait en effet concomitante d'une crue du canal Ismaïlia, qui a déversé ses eaux douces dans le lac d'eau salée Timsah (CAMT, 1898). De nombreux hygiénistes condamnent alors comme essentiellement nuisibles les mélanges d'eau douce et d'eau salée à proximité d'une ville. Le rapport des médecins de la Compagnie attribue I'épidémie en grande partie au mélange des eaux, travaux d'hygiénistes européens à l'appui (CAMT, 1887). En août 1887, les travaux de barrage de la Compagnie font cesser ce mélange. Ils sont considérés comme un véritable progrès de la science épidémiologique pour la salubrité de la ville. Ferdinand de Lesseps lit à l'Académie des sciences le rapport médical explicitant « les causes positives de cette apparition [des fièvres] et les moyens par lesquels elle a été victorieusement combattue » ("Les fièvres à Ismaïlia », Revue-Gazette maritime et commerciale, 25 novembre 1887). Pourtant le mal persiste.

En 1897, la Compagnie forme une commission spéciale chargée d'examiner les causes de paludisme et de rechercher les moyens d'y remédier (CAMT, 1897). Persuadée du mauvais effet des infiltrations d'eau, elle recommande en 1899 l'exécution de nouveaux travaux d'assainissement ainsi que le remplacement des fosses non étanches par des tinettes étanches et vidangeables dans un dépotoir éloigné de la ville (CMT, 1899b). Mais si les travaux menés 
bon train participent à l'assainissement de la ville, leur efficacité est sans commune mesure avec celle permise par la découverte des agents véhiculant la maladie : les anophèles.

À partir de 1900, les médecins de la Compagnie assistent aux nouvelles découvertes. Celle de l'hématozoaire du paludisme par Alphonse Laveran qui, médecin militaire à I'hôpital de Constantine, démontre en 1880 que la malaria est due à un parasite des globules rouges du sang. Celle du Major Ronald Ross, de l'École de médecine tropicale de Liverpool, médecin militaire anglais de l'armée des Indes qui identifie le parasite chez le moustique. Grassi, enfin, démontre quant à lui que seule une espèce de moustiques, les anophèles, est parasitée. En 1902, Ronald Ross est invité à venir étudier la situation à Ismaïlia, tandis que le docteur Pressat d'Ismaïlia part étudier les méthodes utilisées dans les Marais pontins contre les moustiques. Ces différents travaux aboutissent à la suppression systématique des eaux stagnantes où se développent les larves (Le Canal de Suez, 22 mars 1904)². Ross conseille d'organiser la destruction des moustiques par le système des « mosquito-brigades » qu'il avait déjà instituées en Sierra Leone, consistant en une brigade de quatre hommes qui passe chaque semaine dans chaque maison pour déposer une couche d'huile de pétrole à la surface des eaux (Pressat, 1904). Le docteur Pressat prescrit en outre, suite à une expérience concluante sur ses effets préventifs, un traitement de tous les agents et ouvriers de la Compagnie par la quinine (Ibid.). Le rapport du président de la Compagnie sur son inspection en Égypte, en 1904, annonce enfin un bilan positif. Les travaux de destruction des moustiques ont obtenu de bons résultats et la fièvre paludéenne a disparu. Le président assure que "les embellissements successifs d'Ismaïlia et la disparition de la fièvre en ont fait non seulement un endroit unique en Égypte mais auquel bien peu de localités pourraient être comparées »(CAMT, 1904). Les résultats bientôt largement diffusés de cette expérience, tant à l'Académie des sciences par le président de la Compagnie que dans les revues médicales du monde entier, décident le gouvernement égyptien à appliquer à Port-Saïd et Suez les mêmes mesures (Tillier, 1908). L'efficacité du comblement des mares stagnantes mise en évidence par le rapport de Ronald Ross en 1903 pousse également le gouvernement à entreprendre en 1905 des travaux comparables en Basse-Égypte (Jagailloux, 1986, p. 136).

Il est frappant d'observer que ce sont les situations hors d'Europe qui ont poussé les savants à continuer leurs recherches sur le paludisme. Les travaux publics et I'amélioration des conditions sanitaires et de I'hygiène avaient eu tendance à faire oublier la maladie en Europe, entraînant ce que Sabine Barles appelle « I'anticipation d'une disparition » (Barles, 1999, p. 307).

2. Note lue par le prince Auguste d'Arenberg à l'Académie des sciences, séance du 14 mars 1904 : «Expérience faite à Ismaïlia pour la suppression du paludisme par la destruction des moustiques », Le Canal de Suez, 22 mars 1904. 


\section{LES THÉORIES HYGIÉNISTES À L'OEUVRE}

La lutte contre les épidémies menée dans la zone du canal de Suez fut non seulement à l'origine de progrès dans la prophylaxie du paludisme mais aussi dans des domaines tels que l'urbanisme et l'architecture. Fondées ex nihilo ou développées à partir d'un petit noyau existant, les villes du Canal ont permis I'application des principes hygiénistes développés au cours du XIX ${ }^{\mathrm{e}}$ siècle en Europe. C'est en Grande-Bretagne, première puissance industrielle mondiale et premier pays à prendre des mesures sanitaires, qu'Edwin Chadwick (18001890), suite à l'étude des causes de la surmortalité des populations urbaines en 1842, préconise quatre solutions appelées à se généraliser dans l'Europe entière, consistant à paver les rues, à construire des égouts couverts, à développer I'aération des logements et I'adduction d'eau (Pinol, 1991, p. 47). Dans les villes du Canal, ces principes sont systématiquement appliqués alors qu'ils ne sont mis en œuvre que progressivement dans les villes européennes où il est plus difficile de développer de nouveaux réseaux à travers la trame urbaine existante.

\section{Une priorité : la mise en place des réseaux d'eaux}

L'assainissement et la distribution d'eau potable sont les premiers garants de la salubrité des villes du Canal. L'adduction d'eau, une priorité dans ces villes du désert, est rapidement assurée par la Compagnie. La situation sanitaire $d^{\prime}$ Ismaïlia en particulier nécessite des systèmes d'alimentation en eau douce les plus modernes, constamment améliorés. Après avoir été apportée pendant quelques années par un canal, l'eau est ensuite distribuée par des conduites en fonte et des béliers hydrauliques, système inauguré en 1880. Avec la réapparition de la malaria en 1897, la Compagnie décide d'établir une distribution d'eau filtrée. Une nouvelle usine est alors installée à l'ouest de la ville. La canalisation d'eau brute est toutefois conservée pour l'arrosage des jardins (CAMT, 1910).

Au cours de son existence, la Compagnie reste constamment à la pointe des progrès techniques en matière d'assainissement. L'étude de la propagation de l'épidémie de choléra de 1947 en Égypte est révélatrice à cet égard. La zone du Canal semble en effet bénéficier d'une immunité alors que l'épidémie se répand dans les provinces du Delta. L'architecte Claude Chabal attribue au système de filtration d'eau potable cet effet. II vante dans un rapport de 1948 le système utilisé dans les trois grandes villes du Canal, dont son père fut l'inventeur et qu'il recommande à toutes les villes d'Égypte (CAMT, 1948). Chaque station d'épuration des eaux est constituée de plusieurs bassins munis de matériaux de filtration de plus en plus fins. L'eau passant d'un stade à l'autre par déversement profite d'une exposition à l'air et à la lumière et ainsi d'une purification bactériologique supplémentaire (Royer, 1932, p. 208). Ce système 
d'épuration, utilisé à Londres et à Paris, a été perfectionné par les ingénieurs français Puech et Chabal, créateurs de l'École française de Filtration.

Un autre élément important de la salubrité des villes de l'isthme est la création des réseaux d'égouts. Dans les grandes villes européennes, ils existent et se développent depuis le début du xix siècle. Mais en France, en 1892, seules 90 villes sur 691 disposent d'égouts (Pinol, 1991, p. 195).

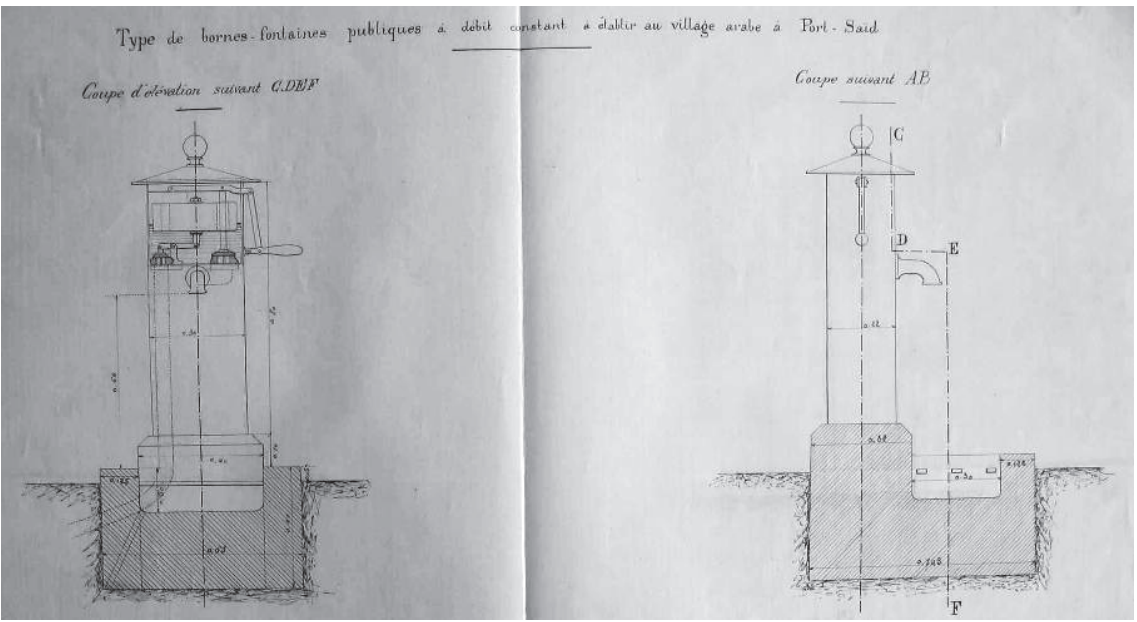

Type de borne fontaine. CAMT 19950604349

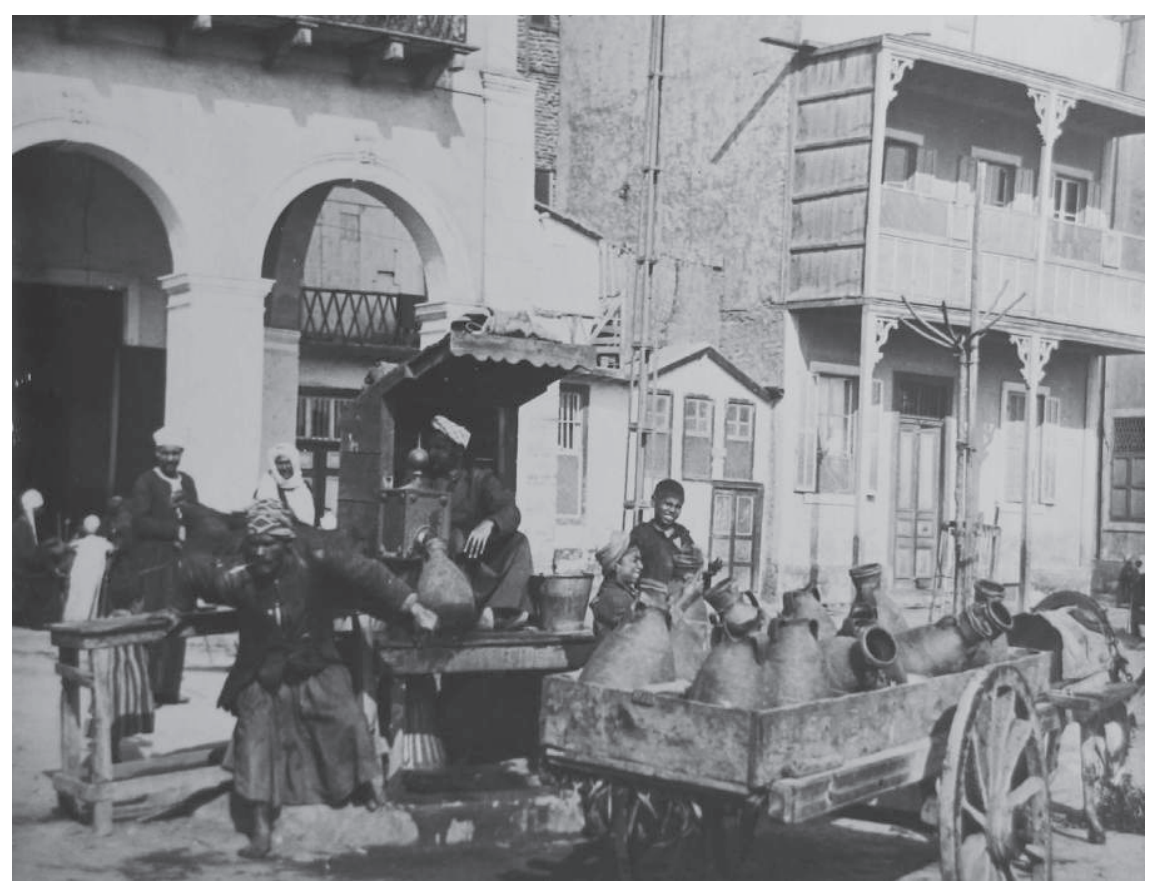

Borne fontaine rue Muhammad 'Alî à Port-Saïd, c. 1910. CAMT 2000036484 
Ce sont les ingénieurs qui ont relayé sur le terrain les théories des médecins hygiénistes et imposé les réseaux d'égouts. Alfred DurandClaye (v. 1840-1888), président de la Commission technique de I'assainissement de Paris, ancien élève de l'École des Ponts et Chaussées, défend le principe du tout-à-l'égout et de l'épuration des eaux par le sol. Pour ses services d'Égypte, la Compagnie recrute elle aussi ses cadres parmi les anciens élèves de l'École des Ponts et Chaussées. La première ville du Canal à être équipée est Ismaïlia. À partir de

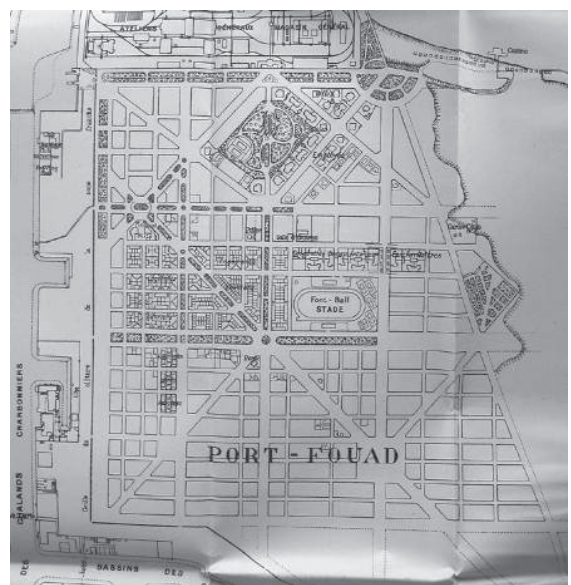

Port-Fouad, travaux d'aménagement et d'extension, 1935. CAMT 19950600056 1907, les ingénieurs de la Compagnie envisagent de la doter d'un système d'égout collecteur. L'ingénieur-conseil Quellenec, issu de l'École des Ponts et Chaussées, est partisan de I'assainissement complet de I'agglomération. Considérant qu'Ismaïlia est à beaucoup d'égards une petite ville modèle, que sa voirie et ses jardins en font la perle de l'Égypte, il estime qu'il serait digne de la Compagnie de contribuer à assainir la ville suivant les règles de I'hygiène moderne (CAMT, 1907). Bien que I'assainissement des villes d'Égypte relève du gouvernement, les finances de ce dernier ne permettant pas d'intervenir partout, c'est la Compagnie qui installe les égouts publics, se limitant toutefois au quartier européen.

À Port-Saïd, le réseau d'égouts est construit par le gouvernement égyptien entre 1913 et 1916, grâce au concours financier, sous forme de prêts, de la Compagnie. Les travaux de raccordement des logements ouvriers sont pris en charge par cette dernière seule.

Enfin à Port-Fouad, cité construite sur la rive Asie du Canal face à PortSaïd, inaugurée en 1926, la Compagnie a d'abord adopté un système de fosses septiques en attendant que le gouvernement prenne une décision au sujet du statut administratif et financier des surfaces de la nouvelle ville. Toutes les maisons ouvrières de cette admirable cité-jardin sont munies de fosses de type "Mouras ", du nom de l'inventeur français de la fosse septique (brevet de 1881), qui seule permet une liquéfaction des matières fécales par des microbes anaérobies. De là, les eaux usées s'écoulent vers des collecteurs et de là vers le port (CAMT, 1942). Lorsque le déversement des eaux dans le port est interdit par les services sanitaires du gouvernement, les discussions s'ouvrent pour trouver une solution par système d'épandage ou par station d'épuration. 


\section{L'aménagement des villes, une mission partagée}

De l'hygiène publique à l'aménagement de la cité il n'y a qu'un pas, que le docteur Aubert-Roche, comme beaucoup de ses collègues hygiénistes en Europe, souhaite franchir. À son initiative, les médecins de la Compagnie jouent un rôle direct dans l'aménagement des villes. Il entend promouvoir les études du milieu permettant d'analyser les qualités des zones d'implantation de la Compagnie. Ainsi met-il en valeur les effets bénéfiques du climat de la circonscription d'Ismaïlia sur la santé :

La position toute spéciale de cette ville au milieu des sables, sur un lac magnifique, entourée de cultures et de jardins, centre de l'isthme, point de jonction entre l'Égypte et le commerce du monde, fera de cette localité une importante cité comme affaires, richesse et santé. Je ne crois pas qu'il y ait au monde un site plus salubre, pour des bains de mer une plage plus belle que celle du lac Timsah, pour les affections de poitrine un meilleur climat. (CAMT, 1865b)

Le docteur Aubert-Roche indique également comment aménager au mieux, dans le respect des règles hygiénistes de base, la future ville de Port-Tewfik, sur le terre-plein de Suez :

Lorsque les terrains qui se trouvent entre le canal maritime et le chemin de fer se couvriront d'habitations, si, au lieu des rues sales et étroites du Suez actuel, on trace de belles voies de communication où l'air circule librement, où les maisons soient bien espacées, si la propreté y est bien entretenue, si les eaux ménagères et autres s'écoulent au lieu de s'imprégner dans le sol et y croupir, si l'on observe quelque peu, dans les rues, sur les quais et dans les maisons, les règles les plus élémentaires de l'hygiène, que l'eau soit abondante, et c'est très facile, je suis convaincu que la cité qui va se former, outre sa position dans un site des plus grandioses, sera des plus salubres. (CAMT, 1868)

Le médecin-chef fait même des propositions concrètes d'aménagement :

La ville d'Ismailia, construite sur un sol sec et sablonneux se présente sous un aspect assez grandiose ; elle est bien dessinée, mais elle offre un inconvénient, c'est la largeur des rues et l'absence de tout abri contre le soleil. II faut absolument que les grandes rues et la place soient plantées d'arbres. (CAMT, 1865)

En Égypte, ces recommandations avaient été faites notamment après le voyage en Europe du khédive Ismaïl en 1867. La préoccupation hygiéniste est présente dans la dimension symbolique que revêt l'aménagement de la ville de Port-Saïd, porte d'entrée du Canal. Le nouveau gouverneur de la zone, entrant en fonction en 1925, déclare que Port-Saïd étant l'une des principales villes de l'Égypte, une station mondiale pour ceux qui viennent de I'Occident ou de l'Extrême-Orient, doit être, dans son organisation, " modèle de la renaissance de l'Égypte sous le règne de Sa Majesté le roi Fouad ler, le 


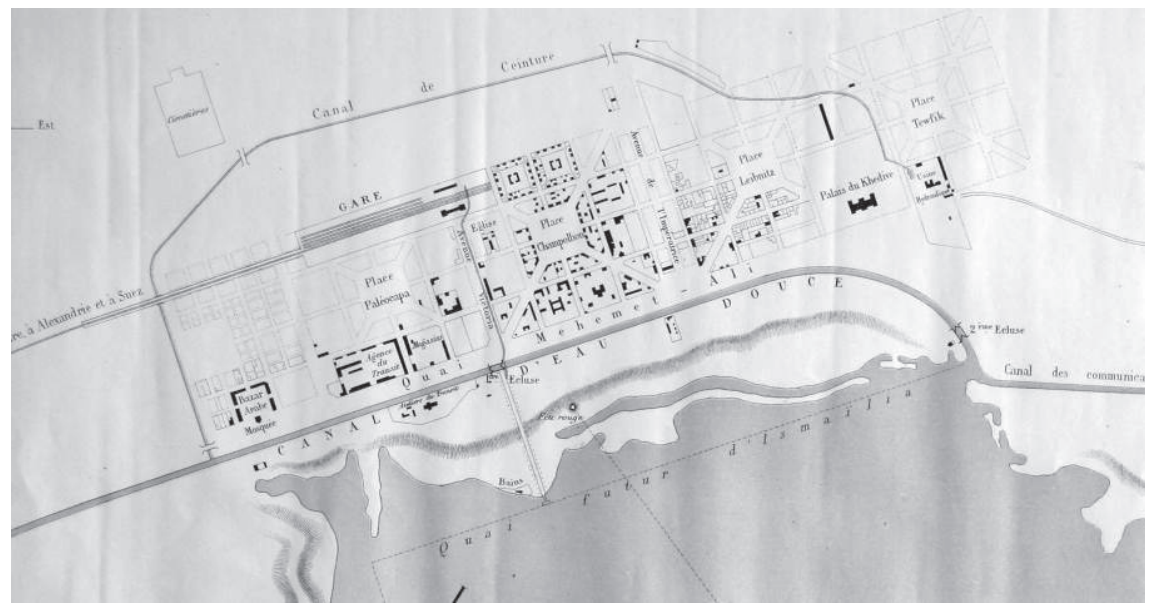

Plan d'Ismaïlia en 1869. CAMT 19950605547

Grand Réformateur, et un exemple du progrès et de la civilisation auxquels l'Égypte aspire dès l'époque du Grand Mohamed Aly et de celle de son petitfils l'auguste Ismaïl»(CAMT, 1925). Le gouverneur souligne l'importance dans cet esprit de la construction de villas d'été et des deux marchés de la ville, mais aussi la pose de macadam dans certaines rues. Cette dernière invention, perçue comme un rempart entre le sol et le sous-sol, se répand depuis le milieu du $x x^{\mathrm{e}}$ siècle.

Les valeurs hygiénistes dominent donc la conception de l'urbanisme des villes de l'isthme. Le chef du service du domaine lui-même justifie sa volonté de diviser le village arabe de Port-Saïd en parcelles régulières parce que « tous les recoins que forme ce groupement irrégulier sont autant de foyers d'infection que I'hygiène commande de faire disparaître dès que cela est possible » (CAMT, 1880).

On retrouve dans toutes les villes du Canal la ségrégation spatiale plus ou moins stricte des groupes, qui est la règle dans les villes coloniales. Il est difficile de déterminer, au vu des sources étudiées ici, comment s'est appliquée cette règle. Selon Zayn al-'Abidîn Nigm, la division de Port-Saïd pourrait s'expliquer à la fois par le désir des indigènes de s'isoler des étrangers afin de conserver leur vie sociale, religieuse et quotidienne, et par la réservation par la Compagnie de certains terrains au bénéfice des seuls Européens (Zayn al-'Abidîn Nigm, 1987).

À Ismaïlia, le plan d'urbanisme conçu dès 1862 consiste en une répétition du même module de base, rendant difficile l'identification a priori d'une ville européenne et d'une ville arabe. En revanche, la manière dont se développera le lotissement fera apparaître l'une et l'autre. Aux alentours de 1920, à Port-Fouad, le découpage par nationalité est un principe d'organisation de la ville que l'ingénieur Paul Solente inscrit au plan d'urbanisme, aux alentours de 1920, en augmentant la largeur de l'avenue située entre le quartier européen et le quartier arabe (Piquet, 2002, p. 175). Les règles de la ségrégation 
spatiale, à l'aune de l'expérience du Maroc et de I'Algérie notamment, sont alors bien définies. Selon I'ancien directeur des Congrès de l'Exposition coloniale, la séparation des « indigènes » et des «blancs » est nécessaire, d'une part parce que les populations locales craignent le contact des Européens, d'autre part pour préserver la santé des Européens (Royer, 1932, p. 11).
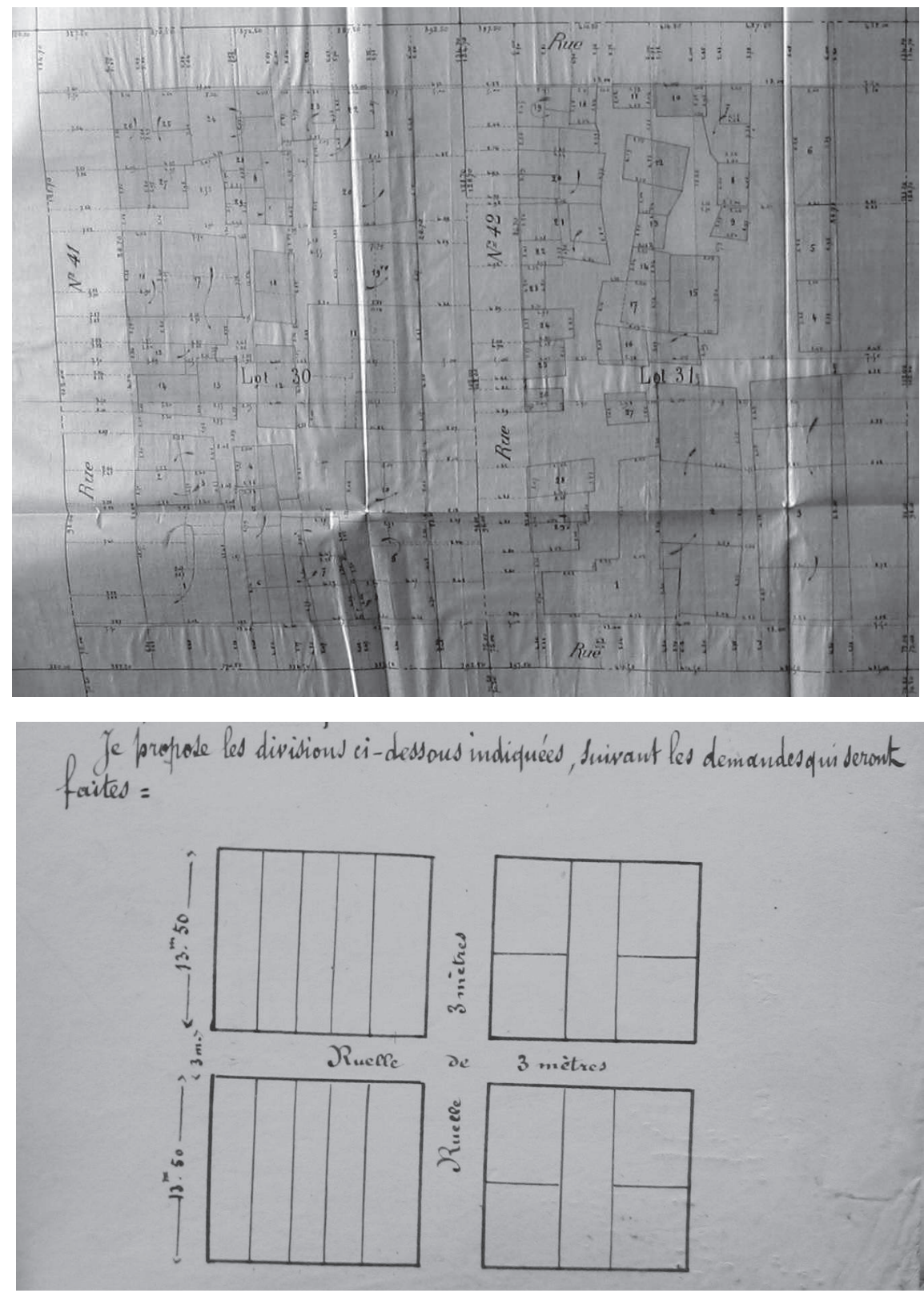

Plan du village arabe de Port-Saïd en 1880 et projet de parcellaire. CAMT 19950603563 
Dans les villes d'Indochine, Ernest Hébrard justifie aussi la ségrégation spatiale pour cette dernière raison (Wright, 1991, p. 221). Cette précaution était déjà mise en avant par le docteur Pressat dans le cadre de ses recherches sur la prophylaxie du paludisme (Pressat, 1904). Par contre, Ronald Ross la considérait peu utile au regard du mode de propagation de la maladie par les moustiques (CAMT, 1902).

Quelles que soient les raisons de son existence, le village arabe de PortSaïd est placé comme la ville européenne sous la coupe des services de la Compagnie et fait l'objet d'un plan visant à améliorer son entretien. En imposant des ruelles de trois mètres de large, le chef du service du domaine entend exiger des habitants qu'ils y déposent leurs ordures pour que les agents de la voirie puissent les enlever. S'il impose les vues des Occidentaux concernant I'aménagement urbain, c'est sous couvert d'hygiénisme : «En outre, il faut obliger les indigènes, dans leur propre intérêt, à disposer leurs installations de manière à se ménager des courants d'air qui assainissent leurs habitations, ce qui sera facile avec des parcelles qui auront façade sur la rue et débouché sur la ruelle $»$.

Toutes les instances, qu'elles soient privées ou publiques, vont dans le sens d'une exaltation des règles de l'hygiène moderne. À Port-Saïd, le gouverneur du Canal, représentant du gouvernement égyptien, exige que les nouvelles constructions soient desservies le plus rapidement possible par des chaussées. Il attire l'attention de la commission du domaine commun sur les inconvénients qui découlent de cette lacune, tant au point de vue de la circulation qu'au point de vue hygiénique, l'absence de chaussée empêchant les équipes de la municipalité de procéder facilement au nettoyage de ces quartiers. Le service sanitaire du gouvernement intervient également dans I'amélioration des conditions hygiéniques des villes. Non seulement le projet d'aménagement du quartier du vieux Manakh à Port-Saïd comprend la substitution des maisons en bois par des maisons en maçonnerie, mais il contraint aussi les locataires à reconstruire leurs étables séparées des habitations. Le service sanitaire subdivise le quartier en cinq secteurs : habitations, étables pour vaches et buffles, écuries pour chevaux, étables pour chèvres et porcheries (CAMT, 1924).

Si rues larges et parcelles régulières participent d'un urbanisme moderne, il en va de même des espaces verts. La reconnaissance de leur intérêt en prophylaxie en a fait depuis Haussmann les " poumons verts » des villes. Le principe énoncé par Koch suivant lequel l'air pur et la lumière sont des armes efficaces contre la maladie, serait partout respecté (Charvet, 2005, p. 81). Pour le lotissement du quartier au sud de la rue $n^{\circ} 99$ à Port-Saïd, la délégation présente un projet comportant des places publiques, des parcs et des rues d'une largeur d'au moins dix mètres afin que ce quartier soit des plus modernes et des plus sains. Un élu « indigène » de la commission municipale déclare lui-même : « [...] Cette considération est celle qui doit prévaloir et là il nous suffit pour nous en rendre compte de voir les défauts du quartier indigène. Les rues larges 
et l'existence de places publiques rendent les maisons saines grâce à leur aération et leur exposition au soleil »(CAMT, 1930a).

\section{L'architecture, un facteur de santé}

L'étude des conditions climatiques a non seulement des répercussions sur I'établissement des camps et l'urbanisme des villes naissantes mais aussi sur les constructions. L'adaptation aux conditions météorologiques serait favorisée par des habitations bien situées autant que bien construites, dans une localité bien choisie. Ce sont les médecins qui indiquent les dispositions et l'orientation à donner aux baraques, tentes et autres modes de logement : "qu'ils soient élevés le plus possible au-dessus du sol, que l'air y circule facilement, qu'ils soient bien clos la nuit. II faut que les habitations soient fraîches pendant le jour, qu'elles garantissent de l'humidité des nuits et des variations brusques de la température » (CAMT, 1865). Certes la rapidité des constructions, nécessitée par l'afflux de population, ne permet guère de respecter dès le début toutes ces précautions. Rappelons qu'à Ismaïlia, il n'y avait pas une seule maison en avril 1862 alors qu'à peine trois ans plus tard le site est devenu une ville, le centre administratif des travaux, avec une population de 2000 âmes environ.

La vigilance des médecins dans l'isthme permettrait tout de même d'observer progressivement une amélioration notable de I'habitat. Les logements

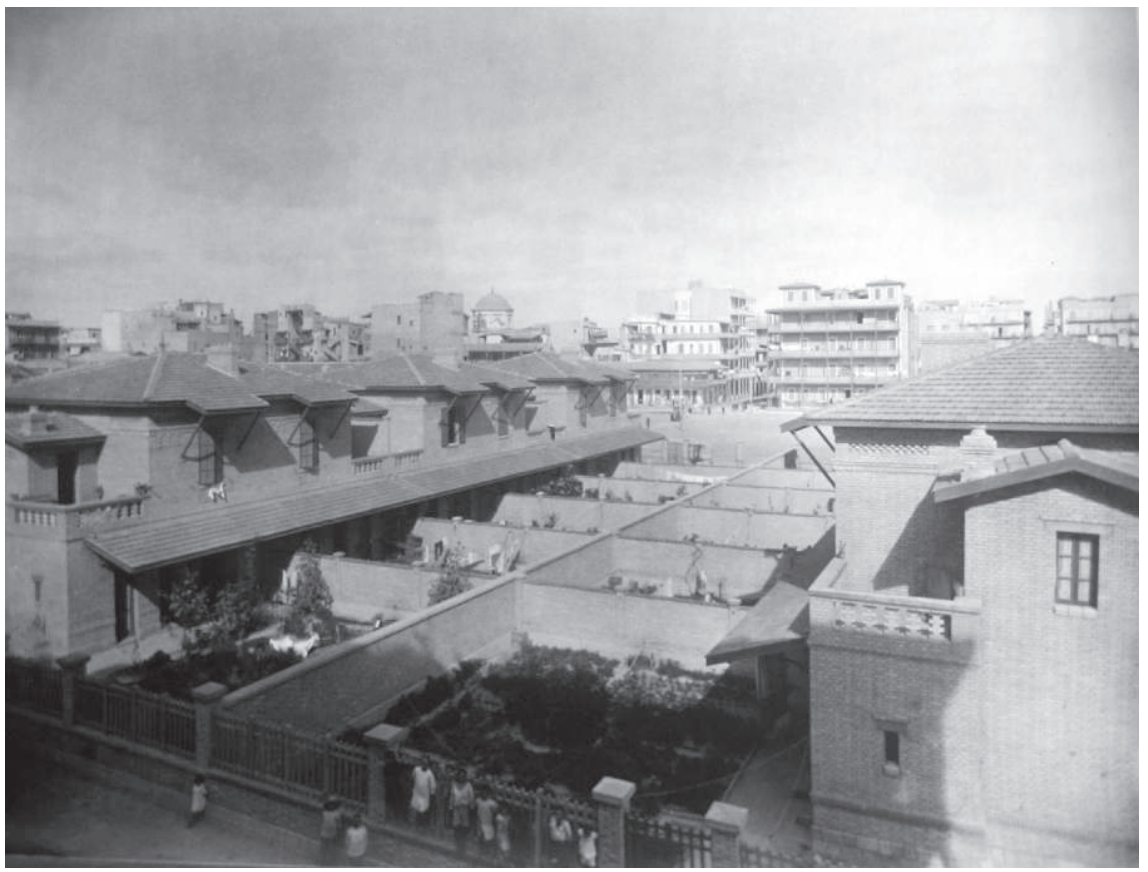

Logements pour ouvriers européens rive Afrique à Port-Saïd. CAMT 2000036484 
deviennent plus propres et les modes de construction efficaces contre l'abaissement de température la nuit et contre la chaleur le jour. Les premières constructions en nattes recouvertes de mortier, en bois ou en briques crues laissent rapidement place à des constructions plus définitives en briques ou pierre, couvertes de tuiles. Les conseils du docteur Aubert-Roche mainte fois réitérés sont peu à peu mis en pratique. II rappelle dans son rapport de 1869 que I'habitation dans l'isthme mérite une attention toute spéciale, qu'elle est avec I'alimentation la base matérielle de la santé. Selon lui, une bonne habitation agit autant sur le moral que sur le physique, et rend les précautions et les soins hygiéniques plus faciles. Ses conseils concernent la construction elle-même :

L'Européen qui voudra habiter dans l'isthme devra donc faire attention à ce que sa maison soit le plus possible exposée au Nord [...] ; que les murailles aient au moins $50 \mathrm{~cm}$ d'épaisseur ; qu'elle soit entourée d'une véranda et de grands arbres afin que le soleil n'échauffe ni le sol ni les murailles; les chambres devront être hautes, bien aérées, à fenêtres opposées, avec persiennes, afin de se rendre maître de la lumière, des courants d'air et de les diriger. (CAMT, 1869)

Très présent pendant les travaux de percement du Canal, époque où les ouvriers restaient logés dans des habitations provisoires, simples toiles de tente ou cabanes en roseaux, le service médical reste actif tout au long du xx siècle, préconisant les meilleures solutions sanitaires lorsque de nouveaux projets s'élaborent. L'ingénieur chef qui présente le projet de quarante-huit logements ouvriers indigènes rive Afrique à Port-Saïd met en avant les avantages hygiéniques de leur conception : "Tant au point de vue sanitaire qu'au point de vue de l'entretien, les meilleures conditions se trouveront réunies »(CAMT, 1919). La nécessité d'une collaboration intime entre les services chargés des constructions et le service médical est rappelée lors d'une conférence des chefs de service en 1922 à propos des logements d'ouvriers construits à Ismaïlia au bâtiment 126 dont les planchers de sapin ont été remplacés par du carrelage de ciment. Le service médical signale qu'il est malsain sous ce climat très humide de coucher dans des pièces carrelées sans sous-sol, qu'il faut considérer la menace d'angines, de bronchites et de rhumatismes (CAMT, 1922). De même, c'est un médecin de la Compagnie qui signale à l'agent supérieur les inconvénients des cabinets d'aisance des gares du Canal. II remarque que la Compagnie, qui a été I'initiatrice de l'adoption des fosses Mouras en Égypte, devenue une règle, doit en faire profiter le personnel des gares (CAMT, 1905).

Les considérations hygiénistes s'étendent au-delà de la construction ellemême. C'est le cas lorsque la Compagnie achète en 1928 un terrain à Port-Saïd

3. Décret de juillet 1888 autorisant la construction dans toutes les rues de Port-Saïd de 15 à 30 mètres. 


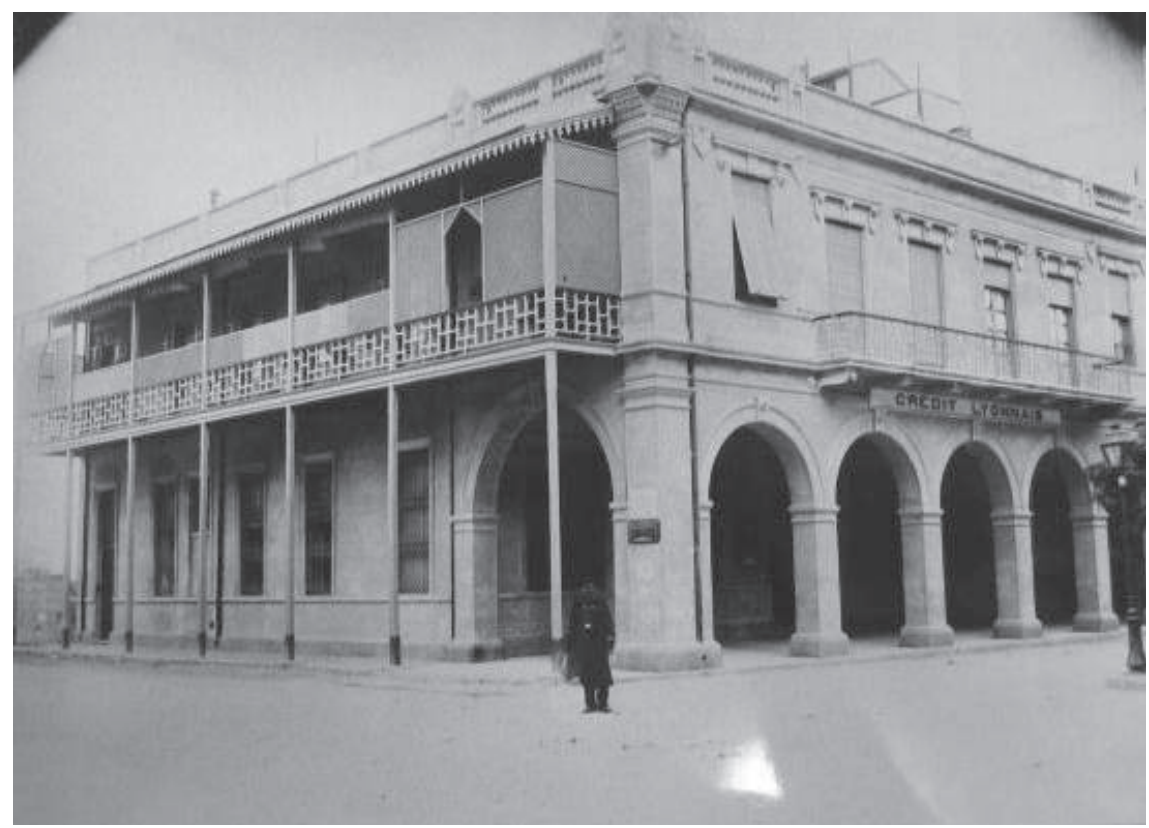

Bâtiment à arcades : I'agence du Crédit lyonnais à Port-Saïd en 1912. Archives historiques de LCL-Le Crédit lyonnais, P-NB-2-0289

simplement en vue d'empêcher sur cette parcelle la construction éventuelle d'un immeuble de plusieurs étages qui priverait d'air et de lumière les maisons ouvrières voisines lui appartenant (CAMT, 1930b).

L'épisode des arcades à Port-Saïd, opposant les «pro-» et les « anti-arcadiens », révèle également l'importance des considérations hygiénistes dans l'architecture des villes du Canal. Il est provoqué par la promulgation par le service du Tanzîm égyptien d'une nouvelle règle de construction ayant trois objectifs : I'embellissement de la ville, la facilité de circulation et la salubrité publique ${ }^{3}$. Or ce dernier aspect est critiqué à bien des égards. Le journal local, Le Phare de Port-Saïd se fait le relais des griefs de ses lecteurs, qui critiquent aussi bien l'esthétique que l'aspect hygiénique. Les arcades sont d'abord accusées d'empêcher la lumière du jour de passer : "Toutes les maisons de Port-Saïd sont faites de telle façon qu'il n'est pas possible d'y construire des arcades sans les réduire à l'état de cavernes non habitables » (Le Phare de PS, 23 février 1889). Ensuite, elles seraient susceptibles de nuire à la circulation de l'air en rétrécissant les rues dont la largeur avait été déterminée à l'origine par le conseil d'hygiène et de salubrité publique « pour la grande gloire et le triomphe des conditions hygiéniques»(Le Phare de PS, 6 avril 1889). Alors que les arcades évoquent à certains le quartier des Tuileries, associant élégance et hygiène, pour d'autres, elles représentent un rétrécissement des rues risquant d'anéantir l'effet bénéfique de l'air : «Avec les arcades, le vent, brisé sur tout son parcours, perdra sa puissance desséchante » (Le Phare de PS, 16 mars 1889). Le combat continuera entre arcadiens et anti-arcadiens jusqu'à 
I'annulation du décret de juillet 1888 mais la construction d'arcades continuera à être autorisée dans les rues où leur construction avait déjà commencé (Le Phare de PS, 27 avril 1889).

\section{CONCLUSION}

Les besoins en main-d'œuvre liés au percement puis à l'exploitation du Canal ont incité la Compagnie universelle du canal maritime de Suez à mettre en place une surveillance sanitaire et un service de santé dans l'isthme. Dans un territoire concédé par le gouvernement qui ne deviendra province égyptienne qu'en 1868, elle installe les fondements d'une véritable politique de santé publique. Ni la fin des travaux ni la mise en place du gouvernorat en 1868 ne signent pour autant la fin de ce service de santé. En association avec le gouvernement ou pour combler les déficiences de ce dernier, il continue de promouvoir l'hygiène, la santé, I'assainissement et I'aménagement des villes créées ou développées dans l'isthme.

La mise en place d'un service de santé par la Compagnie aurait participé à une « acculturation médicale de l'Égypte » (Jagailloux, 1986, p. 8), substituant une médecine à l'occidentale à une médecine traditionnelle non scientifique. Mais le service de santé de la Compagnie a été aussi à l'origine d'un bouleversement des habitudes, en particulier en matière d'assainissement, d'urbanisme et d'habitat.

Bien que l'expertise européenne, particulièrement française, y fût très impliquée, les villes du Canal étaient soumises à une double autorité : celle de la Compagnie, concessionnaire des terrains urbanisés et parfois même chargée de l'édilité publique, celle du gouvernement égyptien, à travers le service de Tanzîm, chargé de la voirie, et le service sanitaire. Ces différentes autorités ont tendu, malgré des frictions passagères concernant la responsabilité et surtout la prise en charge du coût de ces services, à harmoniser leurs actions, au nom de l'Hygiène.

Les vues des Européens en matière d'urbanisme et de construction ont été introduites dans cette partie d'Égypte au nom des théories hygiénistes et des avantages économiques et esthétiques qu'elles sont supposées procurer. Ces théories, véhiculées par les médecins et les ingénieurs de la Compagnie notamment, ne font cependant pas des villes de l'isthme des exemples précurseurs d'un urbanisme moderne. Ces villes ont certes profité d'une application à l'échelle globale de ces théories. Les réseaux ont été beaucoup plus rapidement organisés dans les villes de Port-Saïd, Port-Fouad, Port-Tewfik ou Ismaïlia que dans les grandes villes égyptiennes et la plupart des villes européennes. Au Caire, I'alimentation en eau potable débute en 1886 et le projet de tout-à-l'égout est mis en œuvre à partir de 1907 (Volait, 2005, p. 242). En Espagne, des études des années 1910 attestent des déficiences généralisées du transport d'eau potable et du réseau d'égouts dans les capitales provinciales (Rodriguez Ocana, 2001, p. 318). De même, les 
règlements imposant un urbanisme et une architecture privilégiant le passage de l'air et de la lumière ont été plus strictement appliqués dans cette partie de l'Égypte que dans les campagnes françaises, où il a fallu attendre l'après-guerre 1914-1918 pour voir se diffuser massivement les règles d'une hygiène publique moderne. Nées et développées dans des conditions d'exception, les villes du canal de Suez ont constitué des terrains privilégiés d'application des théories hygiénistes et leur situation particulière a favorisé l'émergence de figures de la santé remarquables en Égypte. Leur urbanisme et leur architecture constituent en outre un pan de l'histoire de l'expertise européenne à l'étranger.

Que ce soit dans le domaine de la prévention, des soins, de l'assainissement, de l'urbanisme ou de l'architecture, les actions menées par la Compagnie dans l'isthme de Suez ont été considérées comme instructives pour la colonisation européenne. Le docteur Pressat publie son ouvrage sur la prophylaxie du paludisme essentiellement pour formuler des conseils en vue des expéditions coloniales. Il affirme : "Ce que nous avons obtenu à Ismaïlia peut être réalisé avec le même succès dans un grand nombre de foyers de paludisme, et nous souhaitons vivement d'en voir tenter l'expérience, en particulier dans nos colonies » (Pressat, 1905, avant-propos). La Compagnie est également représentée lors du Congrès sur l'urbanisme aux colonies organisé dans le cadre de l'Exposition coloniale de 1931 (« Naissance et développement des villes du canal » dans Royer, 1932). Elle y trouve l'occasion de mettre en valeur ses réalisations et de croiser son expertise en matière d'assainissement, d'urbanisme et de construction avec les cadres de l'administration coloniale en poste du Maroc à l'Indochine.

\section{RÉFÉRENCES BIBLIOGRAPHIQUES}

Barles Sabine, 1999, La Ville délétère, médecins et ingénieurs dans l'espace urbain, $x$ VIII ${ }^{e}-x I x^{e}$ siècles, Seyssel, Éd. Champ Vallon.

САMт [Archives nationales, Centre des archives du monde du travail, Roubaix ; fonds de I'Association du Souvenir de Ferdinand de Lesseps et du canal de Suez], 1859a, 1995060 1415, Rapport sur I'organisation du service de santé par le Dr Aubert Roche, médecin en chef, chargé du service de santé, 20 octobre.

- 1859b, $19950601415,1^{\text {er }}$ rapport de santé par M. Zarb, médecin à Port Saïd, 24 mai.

- 1864, 1995060 4134, Lettre de I'agent supérieur au directeur général, 26 juillet.

- 1865a, 1995060 1415, Rapport du médecin-chef Aubert-Roche, $1^{\text {er }}$ juin 1864-1 ${ }^{\text {er }}$ juin 1865.

- 1865b, 1995060 1416, Rapport imprimé sur le service de santé par le Dr Aubert-Roche.

- 1866, 1995060 4134, Rapport sur le choix d'un emplacement pour lazaret provisoire à Port-Saïd, août. 
- 1868, 1995060 1416, Rapport imprimé sur le service de santé par le Dr Aubert-Roche.

- 1869, 1995060 1416, Rapport imprimé sur le service de santé par le Dr Aubert-Roche.

- 1878, 19950660 1428, Lettre de Coulon au président de la Compagnie, 10 novembre.

- 1880, 3563 : Lettre du chef du service du domaine Poilpré au président directeur, 15 avril.

- 1883, 1995060 4134, Communication du Comité de direction, 19 juillet.

- 1884, 1995060 3270, Lemasson, Création d'un hôpital à Port-Saïd, 23 décembre.

- 1885, 1995060 3270, Compte-rendu de réunion du Comité de direction, 24 décembre.

- 1887, 1995060 1428, Rapport des docteurs Machon, Dampeirou, Sini, $1^{\text {er }}$ avril.

- 1890, 1995060 4907, Lettre du chef du service du transit à Ferdinand de Lesseps, 25 août.

- 1897, 19950660 1428, Lettre à Sérionne, $1^{\text {er }}$ septembre.

- 1898, 1995060 1431, Note résumant l'état de la question de la salubrité $\mathrm{d}^{\prime}$ Ismaïlia, $1^{\mathrm{er}}$ septembre.

- 1899a, 1995060 1415, Compte-rendu de la réunion du conseil d'administration, 7 août.

- 1899b, 1995060 1431, Note de la commission médicale sur les travaux à exécuter, janvier.

- 1901, 1995060 3270, Compte-rendu de réunion du Comité de direction, 12 décembre.

- 1902, 1995060 4134, Ronald Ross, Rapport sur la malaria à Ismaïlia et à Suez, 1902.

- 1904, 1995060 1431, Rapport du président, le Prince Auguste d'Arenberg, sur son inspection en Égypte, 1903-1904, Paris, mars.

- 1905, 1995060 4133, Lettre du docteur Camboulin à I'agent supérieur, 4 février.

- 1907, 1995060 4376, Avis de l'ingénieur-conseil Quellennec sur la proposition de I'ingénieur en chef de construire à Ismaïlia un égout collecteur, 6 mai.

- 1910, 1995060 4376, Alimentation en eau douce d'Ismaïlia, 28 juillet.

- 1919, $19950603153,1^{\text {er }}$ Programme de construction de 48 logements à Port-Saïd, proposition de I'ingénieur en chef au Comité de direction, 31 juillet.

- 1922, 1995060 4133, Conférence des chefs de service, 10 février 1922. Annexe 2 : note du service de santé.

- 1924, 1995060 1197, Compte-rendu de la séance de la commission municipale, 7 juillet.

- 1925, 1995060 1197, Séance de la Commission municipale de Port-Saïd, 14 novembre. 
- 1930a, 1995060 1197, Compte-rendu de la séance de la commission municipale, 7 janvier.

- 1930b, 1995060 3153, Compte-rendu de réunion du conseil d'administration, 7 avril

- 1932, 1995060 4166, Paul Nelson, projet pour le pavillon de chirurgie.

- 1938, 1995060 1121, Création d'un Office international d'hygiène publique, Paris, Imprimerie nationale.

- 1942, 1995060 0058, Égouts des villes de l'isthme de Suez, dactylo, mai.

- 1948, 1995060 4134, "Le Choléra en Égypte, immunité de la zone du canal maritime de Suez lors de l'épidémie de choléra de 1947 », par Claude Chabal, architecte DPLG, urbaniste IUP, lauréat de l'institut de technique sanitaire (Société C. Chabal et Cie), dactylo, Paris, 30 avril.

- s.d.1, 1995060 1431, Rapport de la commission sanitaire d'Ismaïlia, dactylo.

- s.d.2, 1995060 1415, Note historique sur I'organisation du service médical dans I'Isthme de 1870 à 1892.

BAeDeKer Karl, 1903, Manuel du voyageur, Leipzig, K. Baedeker.

Charvet Marie, 2005, Les fortifications de Paris. De l'hygiénisme à I'urbanisme, 1880-1919, Rennes, PUR.

Chiffoleau Sylvia, 2001, «L'utopie hygiéniste dans l'Égypte libérale (19201950) » dans Patrice Bourdelais (dir.), Les hygiénistes : enjeux, modèles et pratiques, Paris, Belin.

Claude Viviane, 1999, "Technique sanitaire et réforme urbaine : I'Association générale des hygiénistes et des techniciens municipaux, 1905-1920 » dans Christian Topalov (dir.), Laboratoires du nouveau siècle, la nébuleuse réformatrice et ses réseaux en France 1880-1914, Éd de I'EHESS.

Crosnier-Leconte Marie-Laure, 2006, "Histoires, architectures », dans PortSaïd. Architectures $x x^{e}-x x^{e}$ siècles, Le Caire, IFAO.

Duval Georges, 1981, "L'hôpital moderne 1920-1980», Monuments historiques, $\mathrm{n}^{\circ} 114$, avril-mai.

El Hefnaoui Moustapha, 1986, Les Problèmes contemporains posés par le canal de Suez, Paris, Guillemot, 1951 cité par Serge Jagailloux,1986.

Fijalkow Yankel, 1998, La Construction des îlots insalubres. Paris 1850-1945, Paris, L'Harmattan.

Foucart Bruno, 1981, "Au paradis des hygiénistes », Monuments historiques, $\mathrm{n}^{\circ} 114$, avril-mai.

Garcia Marquez Gabriel, 1987, L'Amour aux temps du choléra, Paris, Grasset, (1 1 ère édition 1985).

Jagailloux Serge, 1986, La Médicalisation de l'Égypte au xix siècle (17981918), Paris, Éd. Recherche sur les civilisations.

Kahn Gustave, 1901, L'Esthétique de la rue, Paris, Bibliothèque Charpentier.

LenOIR Albert et Landry Pierre, 1854, "Théorie des villes. Comment les villes se sont formées ", Revue générale d'Architecture.

Monnerot-Dumaine, 1956, "Les réalisations sanitaires actuelles de la Cie de Suez », La Presse médicale, 21 novembre. 
Montel Nathalie, 1998, "Un chantier médicalement surveillé » dans Le Chantier du canal de Suez (1859-1869). Une histoire des pratiques techniques. Paris, Éditions In Forma/Presses de l'École nationale des Ponts et Chaussées.

Mory Pascal, 2001, "Architecture et hygiénisme à Paris au début du 20 siècle. L'architecte entre savoir médical et pouvoir politique " dans Patrice Bourdelais (dir.), Les hygiénistes : enjeux, modèles et pratiques, Belin.

PINOL Jean-Luc (dir.), 2003, Histoire de l'Europe urbaine, vol II De l'ancien régime à nos jours, expansion et limite d'un modèle, Paris, Le Seuil, 2003.

PINOL Jean-Luc, 1991, Le Monde des villes au xixe siècle, Paris, Hachette.

Piquet Caroline, 2002, "Port-Fouad : New-Harmony dans l'isthme de Suez », Le travail à l'époque contemporaine, $127^{\text {e }}$ congrès des sociétés historiques et scientifiques, Nancy.

Pressat André, 1904, "Prophylaxie du paludisme dans I'isthme de Suez », La Presse médicale, 30 juillet.

- 1905, Le paludisme et les moustiques (prophylaxie), Paris, Masson et Cie.

RASMUSSEN Anne, 2001, "L'hygiène en congrès (1852-1912) : circulation et configuration internationale » dans Patrice Bourdelais (dir.), Les hygiénistes : enjeux, modèles et pratiques, Paris, Belin.

Rodriguez Ocana Esteban, 2001, «Confort, ornementation, hygiène. Modernisation urbaine et hygiène dans l'Espagne du XIx ${ }^{\mathrm{e}}$ siècle » dans Patrice Bourdelais (dir), Les hygiénistes : enjeux, modèles et pratiques, Paris, Belin.

ROYER Jean (dir.), 1932, L'urbanisme aux colonies et dans les pays tropicaux : communications et rapports du Congrès international de l'urbanisme aux colonies et dans les pays de latitude intertropicale (Paris-Vincennes 1015octobre 1931), T. 1.

TILlier Louis [ancien chef du transit de la Compagnie], 1908, « La lutte contre le paludisme à Ismaïlia », La revue du mois, 10 décembre.

VAultier Roger, 1956, "Le canal de Suez et la médecine », La Presse médicale, 21 novembre 1956, n 84

Volait Mercedes, 2005, Architectes et architectures de l'Égypte moderne (1830-1950). Genèse et essor d'une expertise locale, Paris, Maisonneuve et Larose.

WRIGHT Gwendolyn, 1991, The politics of design in French colonial urbanism, Chicago, Chicago University Press.

ZAYN al-'Abidîn Nigm, 1987, Bûr sa'îd, târîkhuhâ wa tatwîruhâ mundhu nacha'tihâ 1859 hattâ 1882 (Port-Saïd, son histoire et son expansion depuis sa création 1859-1882), Le Caire, Al-Hai'a al-'amma al-misriyya li-I-kitâb 